\title{
La métaphore du poisson dans "Poisson d'or" de Le Clézio
}

\section{Par}

\section{Dr/ Ahmed Moawad Abd-Elhadi}

Maître de conférences de littérature française

à la faculté des lettres -El-Arish

Département de français

Université d'El-Arish

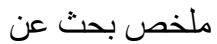

استعاره السمكة في رواية "سمكه من ذهب" للكاتب الروائي "لوكليزيو."



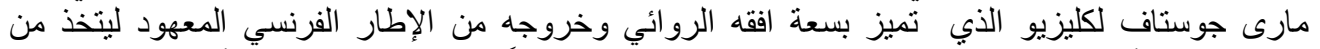

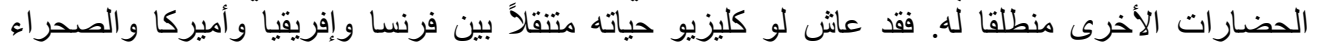

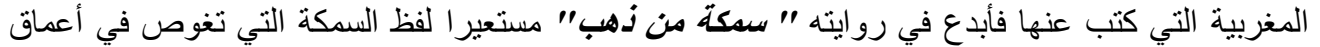

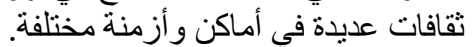

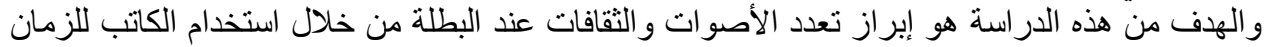

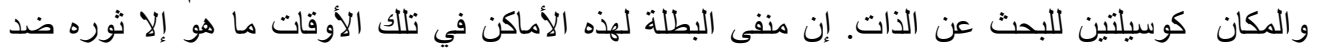

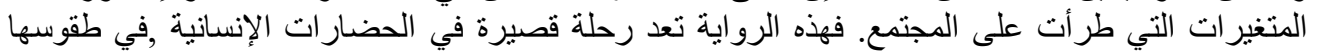

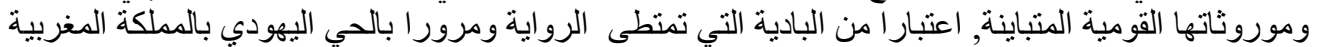

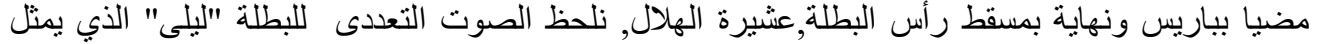

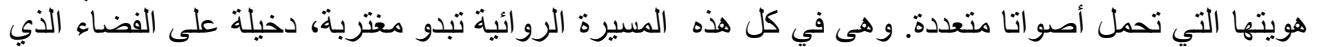

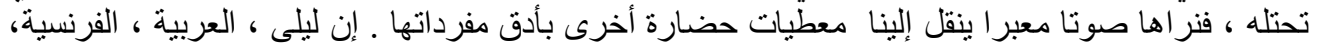

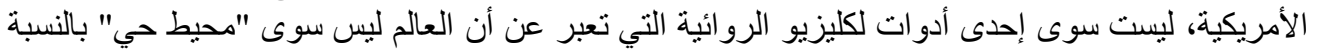

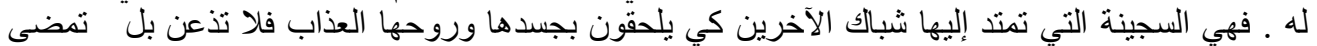
تسخر أدو اتها الطولية في الخروج من قفصها وتنقام شيئا فثيئا حتى تنال حريتها وتجد هو التها.
\end{abstract}

\section{Résumé}

\section{La métaphore du poisson dans "Poisson d'or"} de Le Clézio

L'héroïne de "Poisson d'or" est une exilée africaine vivant à Paris au XXème siècle. Cette étude se concentre sur la diégèse du roman; nous partirons de l'idée de l'exotopie d'après Bakhtine pour traiter comment l'écrivain édifie la plurivocalité de l'héroïne à travers une fonction spécifique du temps et de l'espace narratifs comme un poisson nageant dans beaucoup de cultures et de civilisations différentes. Poisson d'or est un ouvrage polysémique, en particulier multi-temporelle. L'auteur explore des modalités différentes des relations d'un sujet au temps.

Le roman figure la nature humaine et sa complexité à travers la recherche de l'identité de l'héroïne. C'est une révolte contre les contraintes 
sociales que la société dévoile. L'héroïne s'exile pour écrire un texte parlant de son pays dans une vision différente et cette vision donne à son texte une dimension multinationale. Poisson d'or est délimité par les formalités spécifiques du temps et de l'espace, agrandis par l'action du voyage.

Mots-clefs: identité- temps - multiculturel - espace - plurivocalité- Poisson d'or

\section{La métaphore du poisson dans "Poisson d'or" de Le Clézio Introduction:}

Si l'utilisation de noms de lieux pour référer à des individus, des groupes, des évènements est un phénomène très répandu, l'usage de la métaphore ou la métonymie de lieu nous parait plutôt spécial au roman l'identité comme l'assure Jean-Marie Gustave Le Clézio qui raconte, dans le "Poisson d'or", le sort tragique d'une petite fille d'Afrique du Nord. Comme un poisson dans l'eau, Lailla-qui signifie en arabe "la nuit". Une fille noire volée à ses parents, battue et vendue, parcourt la terre à la recherche de ses racines, de sa région, de son identité et réussit à aller jusqu'au bout du monde. En quinze ans, de l'Afrique à Paris, en passant par Marseille, la jeune fille finit par revenir à son point de départ: dans le sable du désert.

L'auteur d'une œuvre n'est présent que dans le tout de l'œuvre. "On ne le trouvera pas dans un quelconque élément détaché de ce tout, et moins qu'ailleurs dans le contenu de l'œuvre, qu'on aura isolé de son tout. L'auteur se trouve là où le contenu et la forme fusionnent, et nous en percevons la présence, avant tout, dans la forme" ${ }^{(1)}$ C'est-à-dire que toute œuvre est issue d'une conscience" qui se caractérise par un dessein artistique plus ou moins polyphonique et à destination d'une autre conscience qui se caractérise par une aptitude plus ou moins développée de compréhension responsive active." (2) Certes, lire Le Clézio, c'est reconnaître la profusion de la création métaphorique.

Nous procédons dans cette étude à démontrer la problématique identitaire que Le Clézio développe dans Poisson d'or: la plurivocalité de l'héroïne en sa relation avec le temps et l'espace, il s'agit de l'entrecroisement de destins et de quêtes qui élisent comme lieu de rencontre privilégié l'espace textuel : le temps d'une écriture. Tous ces espaces s'enchevêtrent dans le récit de Laiila. Ces descriptions, tantôt euphoriques tantôt dysphoriques traduisent, plutôt, une relation bien étroite entre le milieu et les personnages qui s'y meuvent. Elles expriment leur état d'âme. L'héroïne de Le Clézio continue à la recherche de son identité et sa place dans son milieu. Cette recherche est provoquée par un exil, car le sentiment d'étrangeté pousse l'héroïne au voyage et à la découverte d'un autre espace et d'une autre culture que les siens. L'héroïne arrive à Paris, cet espace multiculturel qui fait apparaître un phénomène hybride de l'identité multiple. Nous partirons du concept 
d'exotopie d'après Bakhtine en exposant comment l'écrivain édifie les multiculturelles de l'héroïne à travers une fonction spécifique du temps et de l'espace narratifs.

La problématique de cette étude se propose de découvrir l'identité de l'héroïne à travers l'espace et le temps. Ces deux éléments ne servent jamais de cadre purement extérieur, elles sont étroitement liées et associées à la vie psychique et au drame des personnages. Le titre de l'article, nous a amené à diviser ce travail en deux parties, en suivant la méthode analytique critique. Nous tenterons d'expliquer comment le temps et l'espace, par leurs caractéristiques, offrent pour autant des conditions aussi poétiques, aussi créatrices exprimant le caractère humain et sa démarche intérieure. Nous consacrons la première partie à l'étude au fonctionnement de la temporalité dans la plurivocalité de l'héroïne. Dans la deuxième partie, nous traitons l'influence de l'espace sur la plurivocalité de l'héroïne. Pour cette étude, nous nous sommes appuyé sur les études de Bakhtine, ${ }^{(3)}$ Gaston Bachelard, (4) et Philippe Hamon. ${ }^{(5)}$ Nous présentons cet ouvrage comme une morale, une peinture de la nature humaine dans sa complexité et comme une recherche de l'identité à travers l'exil qui est considéré comme une révolte contre les contraintes sociales que la société du début du $\mathrm{XX}^{\text {ème }}$ siècle dévoilait.

Comme cadre théorique de cet article, nous adoptons le point de vue de Mikhaïl Bakhtine en indiquant que la relation entre l'auteur et son héros exige une distanciation. L'écriture autobiographique permet de revenir à nouveau vers soi: "Le moment initial de mon activité esthétique consiste à m'identifier à l'autre: je dois éprouver -voir et savoir-ce qu'il éprouve, me mettre à sa place, coüncider avec lui [...] et, en tout état de cause, après s'être identifié à autrui, il faut opérer en soi-même [je souligne], regagner sa propre place hors de celui qui souffre, et c'est là seulement que le matériau recueilli à la faveur de l'identification pourra être pensé aux plans éthique, cognitif ou esthétique. "(6) Ce retour vers soi permet à l'héroïne d'être vue par son auteur dans son inégalité. La théorie de la "plurivocalité" de Bakhtine est le point de la création. C'est dans son livre La Poétique de Dostoïevski publié en Russie en 1963 que Mikhaïl Bakhtine a parlé pour la première fois de termes à signification "polyphonique". A partir de l'œuvre de ce grand romancier russe, «il a découvert que l'auteur ne livre pas essentiellement en centaines d'autres et arrive à faire parler les désirs, les opinions, les haines et les amours de ses semblables» (7)

Le choix du titre du roman de Le Clézio est loin d'avoir été laissé au hasard, car Poisson d'or exprime l'atmosphère de l'époque en relation avec la multiculturalité de l'héroïne. Il se situe profondément dans l'espace et le temps, chez Le Clézio, qui sont inévitables. L'un s'anime de l'autre, l'un influence l'autre. Ce qu'explique Charles Grivel dans sa production à l'intérêt romanesque: " L'événement narratif ne se propose que muni de 
toutes ces coordonnées narratives. Sans données temporelles, spatiales, le message narratif ne peut être délivré. " ${ }^{(8)}$ Donc, ce titre ne sert jamais de cadre purement extérieur, il est étroitement lié et associé à la vie psychique de l'héroïne. Le romancier s'attache à nous présenter le cadre spatio-temporel et les relations qui existent entre les personnages principaux, car le texte n'est pas " un phénomène fermé mais il reste ouvert sur le temps et l'espace" (9) Signalons que René Wellek et Austin Warren ont raison de dire que le temps et l'espace du roman" ne sont pas ceux de la réalité. (.....) Même la "tranche de vie" $d u$ naturaliste, sont construites selon certaines conventions artistiques. "(10) pour cela, explorer l'espace et le temps dans une œuvre romanesque, c'est essayer de comprendre celle-ci dans l'interstice qu'autorise une analyse textuelle. Bien plus, c'est lui construire un sens à partir de ces deux structures relevant de la poétique.

\section{La plurivocalité de l'héroïne à travers le temps:}

Un texte littéraire est une analyse combinatoire de signes, c'est-à-dire un ensemble de relations des éléments d'un tout, et une analyse structurale "croit que la partie ne peut se comprendre qu'une fois mise en relation avec le tout ce dont elle fait partie." (11) Le temps est l'un des signes fondateurs d'un roman. Grâce aux marques qu'il offre, le roman procure son sens, c'est-à-dire une conscience et une administration. Nous nous occuperons du temps intérieur où la morphologie poétique de Gunther nous a finalement légué trois temps :" Celui de l'acte de raconter, celui qui est raconté, enfin le temps de la vie. Le premier est un temps chronologique ; c'est un temps de lecture plutôt que d'écriture; on ne mesure que son équivalent spatial qui se compte en nombre de pages et de lignes. "(12) Quant au temps raconté, il est compté en années, en mois, en jours et daté dans l'œuvre même. Enfin, le troisième niveau est déterminé, c'est le récit proprement dit ; il consiste dans la relation d'événements réels ou imaginaires; dans la culture écrite, ce roman est identique au texte narratif se basant sur la métaphore. Cette figure-là est une combinaison de différentes perceptions sensuelles à travers lesquelles nous exprimons une image métaphorique.

La structure de Poisson d'or se compose d'un paradoxe témoignant la nature du temps narratif «de la fiction». Dès le premier mot du roman, l'auteur sait que toute chose est achevée. Mais, le lecteur qui commence dès le point où il ne sait rien de ce qui avait lieu ou qui aura lieu. "L'incipit insère l'ouvre dans un intertexte, on peut y voir une prise de position de l'auteur relativement au genre dans sa dimension historique et esthétique." (13) Le Clézio arrange son roman d'une longueur plus ou moins élevé. Au début de Poisson d'or, on aperçoit une hésitation entre le passé et le présent, le texte commence au milieu des événements de l'enfance qui est pour Le Clézio, le représentant symbolique de l'innocence primitive de l'homme face au monde moderne. L'enfance n'est guère à l'abri des modifications imposées par la 
modernité. Plus les villes s'agrandissent, plus l'identité citadine se cristallise. Le monde romanesque de Le Clézio est dominé par la présence des enfants. Son héroïne est une jeune adolescente, Laila, qui a du mal à s'intégrer dans la société métropolitaine. On peut relever à l'appui la citation suivante de notre corpus:

"Quand j'avais six ou sept ans, j'ai été volée.\{...\} C'est plutôt comme un rêve, un cauchemar lointain, terrible, qui revient certaines nuits, qui me trouble même dans le jour. Il y a cette rue blanche de soleil, poussiéreuse et vide, le ciel bleu, le cri déchirant d'un oiseau noir, et tout à coup des mains d'homme qui me jettent au fond d'un grand sac, et j'étouffe. C'est Lalla Asma qui m'a achetée." (14)

La citation ci-dessus, est une exposition ayant une grande valeur parce qu'elle intègre le lecteur dans un monde inconnu; celui de la fiction et de l'irréel. Elle se compose particulièrement de deux lots : le temps et l'espace. Au début du Poisson d'or, l'auteur commence son exposition par la précision du temps puis de l'espace. Le Clézio est la voix de son héroïne, il introduit son texte par l'identification de l'enfance de son héroïne achetée d'une vieille femme juive qui raconte des contes de pauvres à travers le temps historique dans le roman. Cette vision rétrospective est considérée comme une référence temporelle réelle auxquelles se rapportent les événements du roman qui situe l'histoire " qu'il raconte dans un cadre spatio-temporel où tous les composants trouveront leur place, les uns à côté des autres, ou les uns après les autres." (15)

À travers le temps, la culture de l'héroïne s'attache à son passé, son enfance, son origine et son présent comme un poisson qui nageait dans ses mers. Elle ne peut pas oublier sa faiblesse contre la force dominante de l'homme; cette situation a semblé comme un cauchemar terrible. L'héroïne est toujours obsédée par l'acte de son vol employant le présent indicatif dans les verbes:(voler, jeter, étouffer) pour indiquer que la souffrance durera avec le temps sans fin, c'est-à-dire que l'emploi des expressions du temps reflète la douleur de l'héroïne: tout à coup, au fond d'un grand sac, c'est plutôt comme un rêve, un cauchemar lointain, terrible,... etc. D'ailleurs, l'espace joue un rôle très important, l'usage de l'expression" des mains d'homme qui me jettent au fond d'un grand sac" indique la faiblesse de l'héroïne et l'expression " grand sac" montre que l'héroïne est très fragile et très maigre. Le Clézio utilise la comparaison pour achever l'image littéraire: il parle d'une petite fille âgée qui a six ans contre un grand homme qui a des mains fortes, du grand sac. Cette comparaison dévoile l'identité et la culture de l'héroïne dès le début du 
roman, c'est une métaphore où l'auteur a exprimé le petit poisson nageant contre le courant comme une fille résiste contre la violence" j'ai été volé", elle voudrait oublier ces souvenirs terribles, mais elle ne peut pas oublier son enfance qui détermine son avenir. Elle voudrait sortir de son réel à l'autofiction par l'usage un aspect magique en souhaitant que sa vie passée devienne un rêve. On peut citer les expressions: "Il y a cette rue blanche de soleil, poussiéreuse et vide," qui signifient la voix terrible, l'expression ; le cri déchirant d'un oiseau noir" présente une pensée très sombre chez l'héroïne. Cette expression renouvelée à la mémoire de l'héroïne où elle narre: " J'ai réussi à me faufiler par la grande porte bleue qui était restée entrouverte. Puis je me suis mise à courir dans les rues, comme le jour où j'étais allée quérir la sage-femme. J'avais très peur qu'ils ne me rattrapent et me mettent en prison pour avoir laissé mourir Lalla Asma." (16) C'est un aspect de son identité qui a hésité entre le passé et le présent. L'héroïne se souvient toujours ce jour où elle est volée de sa famille. En ce jour de malheur, elle craignait de la prison et le grand sac. C'est-à-dire que le temps influe sur sa vie et sur son identité. Citons à l'appui cette citation:

"C'est comme cela que j'ai quitté sans retour la maison du mellah. Je n'avais rien, pas un sou, j'étais pieds nus avec ma vieille robe, et je n'avais même pas la paire de boucles d'oreilles en or, mes croissants de Lune Hilal, que Lalla Asma avait promis de me laisser en mourant.» (17)

Dans la citation ci-dessus, il y a un autre aspect temporel qui présente une plurivocalité dans la vie de l'héroïne; elle fuit après la mort de la dame juive pour affronter un circuit de souffrance, elle refuse la violence et décide de quitter la maison sans retour, donc, l'auteur précise une autre coté de l'identité de l'héroöne où cette petite fille n'acceptant jamais l'injustice; déclare la recherche de la liberté et la justice dans un autre espace, malgré qu'elle n'a rien, pas un sou, nue avec sa vieille robe.

Les indices temporels assignent à l'histoire une durée. Les moments précis renvoient à des idées fixes qui mettent en place un repérage dans l'absolu auxquels font retourner les indications de la fiction. Ainsi, nous pouvons voir dans Poisson d'or, une durée historique à la vie de l'héroïne pour nous rappeler son passé comme une étape de son identité et pour évoquer sa culture française et arabe qui occupe une place unique dans sa vie tout entière en affirmant une autre forme de plurivocalité : " Le fondouk était bien différent de tout ce que j'avais connu jusqu'alors. C'était une maison ouverte aux quatre vents, située dans une rue passante encombrée de camionnettes, d'autos, de motocyclettes... " (18) L'écrivain raconte les 
événements chronologiquement quand l'héroïne a été volée à six ans. Puis elle est allée au fondouk bien connu. Ensuite, elle est allée à Paris magnifique par ses rues, ses magasins, ses cultures, ses jours, ses nuits, et ses quartiers. Aussi, elle est partie à Boston. Enfin, elle a fait le dernier voyage à sa terre natale en Afrique où elle y retournera plus tard, avec un autre nom, un autre visage, une autre identité, une autre nationalité et une autre culture: " Avant de partir, j'ai touché la main de la vieille femme, lisse et dure comme une pierre du fond de la mer, une seule fois, légèrement, pour ne pas oublier. "(19) Si l'on considère la structure du roman, on constate que les moments sont situés les uns par rapport aux autres dans des enchaînements assez voyants et un peu vagues :

L'importance du temps dans la narration est soulignée dès le début du roman. La trame narrative de "Poisson d'or" s'ouvre d'ailleurs, nous l'avons remarqué, avec la spécification spatio-temporelle. Ce roman nous donne l'impression qu'il n'est qu'une "tranche de vie " (20) de l'hérö̈ne. L'auteur a consacré une grande partie à une période historique pour faire revivre le passé à travers la figure de l'enfance de l'héroïne. La liaison avec le réel se fait par le biais de différents indices. Poisson d'or se sert de personnages référentiels apparaissant dans le texte au milieu des personnages fictifs, d'événements historiques précis et d'indications spatiales absolues. Selon la citation suivante :

" Dans cet endroit, je pouvais parler avec Lalla Asma. Je n'ai jamais su où elle avait été enterrée. $\{\ldots\}$ je sentais que dans ce cimetière j'étais tout près d'elle, qu'elle pouvait m'entendre.je lui racontais ma vie. \{...\} "Grand-mère, vous ne seriez pas fière de moi. Vous qui m'avez toujours dit qu'il faut respecter le bien d'autrui et dire la vérité, voilà que maintenant je suis la plus grande voleuse et la plus grande menteuse de la terre. "》 (21)

Le Clézio offre toujours une personne référentielle à l'héroïne, c'est Lalla Asma qui était une conseillère dans sa vie, elle la consulte non seulement dans sa vie, mais aussi après sa mort. Dans cette citation, il y a un aspect magique de sa culture et sa civilisation où l'héroïne va à la dame juive après sa mort pour lui parler.

Poisson d'or est considéré non seulement comme un modèle de vérité qui marque des indications de dates, mais aussi de nombreuses notions de temps et de durée (mois, jours, heures....): "Un dimanche qu'il pleuvait, il m'a emmenée à la porte Dorée, pour voir le musée des Arts africains. Je crois que je n'étais jamais entrée dans un musée auparavant " ${ }^{(22)}$ Cette citation présente une période réelle dans l'histoire de l'Afrique moderne pendant le voyage de l'hérö̈ne. Le temps chez Le Clézio sert à travers des unités narratives, à ancrer son roman dans le réel.

En effet, la narratrice joue sur un registre très varié pour introduire cette notion du "temps chronique" (23) représenté dans la fiction par des charges 
temporelles à "repérage absolu" ${ }^{(24)}$ L'indication temporelle ne se trouve pas déclarée par les dates indiquées dans Poisson d'or. Le but de l'histoire est la recherche de soi-même au passé. Mais l'histoire peut-elle aller jusqu'à frôler le présent? Dans Poisson d'or, le romancier présente son époque, il propose un tableau complet de sa société, mais il insiste sur les données sociales et non sur les destinées individuelles, il utilise la focalisation interne à travers le premier pronom "je" qui revoie à la narratrice-l'héroïne:

" J'ai lu des livres de géographie, de zoologie, et surtout des romans Nana et germinal de Zola, Madame Bovary et Trois Contes de Flaubert, Les Misérables de Victor Hugo, Une vie de Maupassant, L'étranger et La peste de Camus\{...\} Quand la lumière déclinait, je retournais au Douar Tabriket. Tandis que la barque du passeur glissait sur l'eau pale de l'estuaire, j'avais la tête toute bruissante des mots que je venais de lire, des personnages, des aventures que je venais de vivre." (25)

La multiculturalité de l'héroïne dans ce roman incarne une partie de vie de le Clézio, comme une encyclopédie dans tous les domaines et surtout le domaine de la littérature; elle a lu les chefs-d'œuvre des écrivains français pendant les trois siècles passés, le XVIIIe, XIXe et XXème. En effet, Le Clézio ne cite pas les périodes historiques directement, mais il a laissé son lecteur découvrir les ères à travers les œuvres littéraires mentionnées dans Poisson d'or. Le Clézio a choisi ces ouvrages pour indiquer les périodes historiques de ces auteurs. D'après ces auvres citées, il y a une unité de thème et d'idéologique; c'est la souffrance de l'homme sur la terre. Il y a une osmose entre le sujet et les auteurs mentionnés comme le bovarysme chez madame Bovary qui est considéré comme un courant du réalisme, de même, Victor Hugo qui traite les problèmes de la pauvreté à travers Les Misérables, citons encore "Une vie" le roman célèbre de Maupassant et les œuvres de Camus. Tous ces écrivains mentionnés dans le roman, s'attachent à leurs pays. Donc, cette héroïne a deux identités; européenne et arabique qui apparient de sa culture immense dans tous les domaines scientifiques, comme la géographie, la zoologie et la littérature française et arabe.

Le temps historique se précise dans la citation suivante: "Il (Hakim) parlait du Prophète et de Bilal, son esclave, qui avait le premier lancé l'appel à la prière..." (26) Cette citation montre une autre voix de la culture arabe et islamique chez l'héroïne, cette culture indique une partie de son identité au passé. Donc, cette femme a une identité, non seulement européenne, mais aussi, une identité arabe. Pour Le Clézio, le croisement de cultures dissemblables, dans ce cas la culture française et celle de l'Afrique musulmane, finit en principe en faveur de l'Occident considéré comme moderne et avancé, au détriment de l'autre qui doit avoir la force de conserver son originalité face à un courant urbain basculant toute l'identité. 
L'écrivain utilise le temps historique dans Poisson d'or pour présenter à son lecteur une image plus proche. La tâche qui consiste à préciser le temps historique est difficile. Aucune date n'est stipulée, "L'inscription dans le temps peut être imprécise, mais elle est nécessaire." (27) Ce roman ne se situe pas dans une période historique déterminée où les signes d'actualité sont généralement gommés, mais les événements du texte indiquent la période historique. L'écrivain présente une période réelle dans l'histoire de la France, il expose cette image pour indiquer la valeur de la liberté et de la justice. C'est ainsi que la présence des faits historiques dans ce roman rappelle le temps de l'union, le temps de l'amour chez les Africains, le temps du sacrifice, le temps de la liberté et de la justice. C'est-à-dire que Poisson d'or est un ouvrage polysémique, en particulier multi-temporel. L'auteur explore différentes modalités d'un sujet au temps.

Si la narratrice fournit à l'histoire qu'elle formule des dates précises, c'est parce que "dater et indiquer le passé comme date du texte revient, (....) à le vraisemblabiliser. A garantir dans le texte la vérité de la fiction." (28) Ainsi, le lecteur considère le récit pour vrai, et non pas pour une création fantaisiste. Par ailleurs, en interrogeant Poisson d'or, nous constatons que ces indications temporelles absolues servent, dans la plupart des cas, à annoncer qu'un événement extrêmement important va ou vient d'arriver. Poisson d'or est fondé sur la rupture et le retour en arrière, comme les contes de Hakim autour de l'hérö̈ne et de soi-même, qui présentent un grand type d'anachronismes narratifs, c'est l'analépse du texte. Ces événements constituent un élément essentiel quant à la réception de l'œuvre en tant que biographie :

" Au dernier instant, j'ai vu son regard, et j'ai eu peur, parce que j'ai reconnu les yeux durs, perçant, d'Abel, quand il était entré dans la buanderie. Mais c'était trop tard. Il m'a attrapée par les poignets, il m'a serrée avec une force incroyable, sans dire un mot." (29)

Le Clézio place Poisson d'or dans un temps passé, représentant au lecteur une réalité historique qui lui permet "de retrouver avec précision les événements qui servent de points de repères historique."(30) Cependant, le temps exposé dans Poisson d'or a un caractère complexe et corrompu. L'héroïne de Le Clézio est étrangère au premier abord, mais elle ne l'est qu'en apparence. Elle réfléchit souvent à l'avenir de l'humanité, du monde et de la civilisation, comme on peut le remarquer dans le passage suivant:

" On s'asseyait dans le cafés et on parlait. Hakim était grand et mince, toujours élégant dans son costume noir. Il racontait des choses étranges. Un jour, il m'a apporté un petit livre usé, qui avait été lu par des quantités de mains graisseuses. Ça s'appelait Les Damnés de la terre, et l'auteur s'appelait 
Frantz Fanon. Hakim m'a donné mystérieusement: "Lis-le, tu comprendras beaucoup de choses. "»" (31)

Dans la citation ci-dessus, la plurivocalité de l'héroïne est exposée à travers l'emploi du temps. Cette héroïne pense fréquemment à la postérité de l'humanité et à la culture du monde. L'auteur mentionne cette citation sans préciser une date d'une manière effective à pousser le lecteur à recherche de cette œuvre et reconnaitre son moral et sa valeur: ce livre qui parle du peuple colonisé et sa valeur la plus essentielle "parce que la plus concrète, c'est d'abord la terre: la terre qui doit assurer le pain et, bien sûr, la dignité. Mais cette dignité n'a rien à voir avec la dignité de la personne humaine. Cette personne humaine idéale, il n'en a jamais entendu parler...» ${ }^{(32)}$ Les Damnés de la Terre est le dernier livre de Frantz Fanon, publié quelques jours avant sa mort aux Éditions Maspero en 1961 et traduit en quinze langues. Cette tentative analytique se penche sur le colonialisme, l'aliénation du colonisé et les guerres de libération. Il étudie le rôle que joue la violence entre colonisateur et colonisé. Le livre expose aussi les contradictions essentielles dans l'exercice du pouvoir dans l'ère post-coloniale en Afrique. Fanon met dans ce livre une règle générale: que l'indigène apprend, c'est à rester à sa place, à ne pas dépasser les limites; c'est pourquoi les rêves de l'indigène sont des rêves musculaires, des rêves d'action, des rêves agressifs. En effet, après avoir lu ce livre, l'héroïne rêve qu'elle nage, court, franchit le fleuve d'un pas. La citation ci-dessus confirme cette idée :

"En français, l'examinatrice ne voulait pas croire que j'étais libre. Elle examinait mon passeport, elle regardait mon dossier, et elle disait : "Cessez de me mentir. Où avez-vous fait vos études?" et puis: "Où est votre liste?" Enfin, comme si elle avait honte de s'être mise en colère, elle m'a dit: "Sur qui voulez-vous faire votre explication?" J'ai dit, sans hésiter:" Aimé Césaire. "Ça n'était pas au programme, mais elle était étonnée, elle m'a dit:" eh bien, je vous écoute." J'ai récité par cœur le passage de Cahiers d'un retour du pays natal cité par Frantz Fanon."." (33)

Dans cette citation, l'héroïne a un sentiment d'appartenance à son passé et son espace. C'est son pays perdu, c'est sa mémoire, son bonheur d'enfant, sa plus grande liberté. Ce lieu est le berceau de tous ses rêves. La recherche d'un pays perdu offre à l'écrivain un certain nombre de sentiments de l'enfance. Dans ce roman, il s'agit pour la narratrice de reconstruire la cohérence spatiale du monde pour y inclure: son propre pays.

La vision catastrophique de l'avenir de la narratrice est racontée avec le ton scandaleux ou plutôt, provocateur. Son héroïne est une femme ordinaire sans emploi fixe, une vagabonde, une pauvre, dépendant de la bonne volonté de ses amis qui lui donnent continuellement de l'argent, un abri. Mais, d'un autre côté, c'est une artiste, une femme libre, réfléchissant sur l'existence et 
l'avenir de l'humanité. Ce caractère double de l'héroïne oblige le lecteur à prendre du recul face à tous les faits ordinaires racontés par l'hérö̈nenarratrice. Selon Bachelard, le monde commence pour l'homme par "une révolution d'âme qui bien souvent remonte à l'enfance ${ }^{\prime 34}$. Il en est de même pour le monde scriptural leclézien qui ancré dans le réel, c'est-à-dire, dans la réalité référentielle, va dévoiler l'omniprésence de l'enfance dans le tissu romanesque. On ajoute que, le romancier doit être porté à écrire sur les premières années de sa vie, où le principal lui a été donné:

"J'ai voulu crier, mais il a appuyé un poing sur mon ventre et il a serré d'un coup, comme s'il voulait me casser en deux, et j'ai perdu le souffle, et je me suis effondrée, les bras et les jambes coupés. J'étais seulement sans force, comme dans un cauchemar. $\{$...\}Il était fort, et habile..... Je ne sais pas combien de temps ça a durée, mais m'a semblé une éternité. Il me semblait que ça ne finirait jamais." (35)

L'auteur dans cette citation présente une image de la faiblesse de l'héroïne où elle ne peut pas se défendre. Cette faiblesse qui influe sur sa personnalité. L'auteur utilise les expressions qui expriment ce sens dans une comparaison entre l'héroïne et l'homme harcelant: " elle voulait crier, elle a perdu le souffle, son bras et ses jambes coupés, elle était sans force, elle est fragile, elle ne sait le temps duré, tandis qu'il a appuyé un poing, il a serré d'un coup, il veut la casser en deux, il est fort, et habile, il donne de grands coups de reins ...etc. L'héroïne vit dans un espace européen qui fait une mutation identitaire qu'on va subir au fur et à mesure que le temps passe lourdement" elle ne sait pas combien de temps ça a durée, mais s'a semblé une éternité. Il me semblait que ça ne finirait jamais." En face d'un courant urbain qui change son identité et sa culture.

Nous observons le parallélisme entre le physique et la métaphysique entre le quotidien et la catastrophique. Le passé et le présent s'entrelacent et dialoguent dans l'écriture à la première personne, qui se base sur la mémoire individuelle. La temporalité chez Le Clézio n'existe que dans sa dimension globale du présent qui se nourrit du passé et se projette sur le futur: "Le récit se développe comme monde clos, suffisant, constitué, exige à la fois local (localité) et temporalité. Il doit dire quand, il doit dire où (qui, quoi). " (36) A la fin de roman, la narratrice a terminé son voyage en retournant à sol natal avec sa plurivocalité, sa multiculturalité et sa nouvelle civilisation :

" Loin, au bout de la rue, devant la dernière maison, là où commence le désert, une vieille femme en noir est assise sur un tabouret, devant la porte vide de sa cour. Son visage n'est pas caché par un voile $\{\ldots\}$ Je me suis assise à côté de la vieille femme. Elle est si petite, si maigre, elle m'arrive à peine à l'épaule, comme une enfant. La rue est vide, écorchée par le 
soleil du désert $\{. .$.$\} Maintenant, je sais que je suis enfin arrivée$ au bout de mon voyage. C'est ici, nulle part ailleurs. La rue blanche comme le sel, les murs immobiles, le cri du corbeau. C'est ici que j'ai été volée, il y a quinze ans, "(37)

Dans cette citation se trouve la clé de la problématique de l'œuvre et la mise en valeur de deux éléments constitutifs du roman, le temps et l'espace. Concernant l'espace, les descriptions de l'espace du désert, qui visent à construire le milieu environnant où agissent et se déplacent les personnages et qui sont loin d'être de simples paysages, nous fournissent des références sur l'intériorité. On touche l'influence du temps à la vie de l'héroïne qui va se rechercher à travers quinze ans. A cette époque, elle veut trouver son âme perdue, tandis que les autres glissent dans la nouvelle société sans retour. On remarque la nostalgie pure chez l'héroïne. C'est une métaphore claire où il $\mathrm{y} a$ une relation étroite entre le titre "Poisson d'or " et la fin du roman qui indique qu'elle arrive à son but. Donc, on peut dire que pendant cette période" les quinze ans" l'héroïne soit comme un poisson qui a été loin de l'eau puis elle retourne à sa mer sans changement, alors, c'est une femme d'or qui revient à sa terre natale après un voyage pénible, comme un voyage de vengeance et un voyage de gloire. Le titre du roman "Poisson d'or" signifie que cette héroïne ne se modifie jamais à travers cette durée comme l'or qui ne perd pas son prix. Donc, l'auteur ne choisit pas son titre par hasard, mais il voudrait assurer la vérité de l'identité pure de cette personne d'origine arabe élevée en Europe.

\section{L'influence de l'espace à la plurivocalité de l'héroïne}

La source de tout conflit réside dans le problème de l'espace, ou plutôt, dans le manque d'espace. Il est vrai que la femme dans la réflexion de la narratrice clézienne erre sans cesse dans des rues de Paris, elle sort comme une nomade pour se balader d'une façon hasardeuse. Cette balade, par son rythme et caractère boulevardiers, lui donne une impression de liberté. Mais, simultanément, le lecteur a souvent l'impression de la médiocrité de l'espace. Les cafés sont emplis et les rues nous étonnement par la confusion infinie de toutes les langues. L'amitié est rare. Dans Poisson d'or, il y a des endroits, des chambres ou des appartements, où vivent des immigrés cachés qui dorment sur le canapé ou par terre, parce qu'il n'y a pas assez de lits pour tous. Cet excès dans l'espace crée une impatience, une espérance silencieuse, une accumulation désagréable qui doit finir par éclater.

Dans Poisson d'or, les lieux présentent non seulement des étapes de l'histoire de la vie vivant dans la mémoire de l'héroïne, mais, ils reflètent la plurivocalité de cette héroïne. Selon P. Louis Rey, " L'espace d'un roman imaginé par l'écrivain met ici des mouvements des pensées, des souvenirs, des désirs qui modèlent le personnage en lui imposant ces bornes. "(38) On considère que l'espace est l'arrière-plan des événements du roman, 
contrairement au temps étant comme les événements eux-mêmes. L'espace, est donc, le cadre où les événements ont lieu. L'espace dans ce roman est présenté sur deux dimensions différentes: une immédiate (celle des rues parisiennes) et une globale, celle qui entoure toute la situation de l'héroïne, de l'exil qui est une situation de l'inversion de la dynamique habituelle: en effet, en se déplaçant à l'étranger, le moi devient "autre", tandis que l'autre demeure en soi. Ce développement implique l'utilisation des imagotypes. A l'autoimage du moi inversé s'ajoutent des hétéro-images de français et d'autres étrangers habitant Paris. Ainsi, surgit " un autre" multiple, qui n'est plus un point de référence unique et homogène au "moi". De plus, cet autre, qui est souvent un étranger, se trouve dans la même situation de déplacement que le "moi", tout en restant différent du "moi" par sa nationalité différente. Tout exil volontaire, comme dans le cas de l'héroïne clézienne, commence par le sentiment de l'étrangeté. On peut relever à l'appui la citation suivante de notre corpus :

"C'est pourquoi je ne connais pas mon vrai nom, celui que ma mère m'a donné à ma naissance, ni le nom de mon père, ni le lieu où je suis née. Tout ce que je sais, c'est ce que m'a dit Lalla Asma, que je suis arrivée chez elle une nuit, et pour cela elle m'a appelée Laila, la nuit. Je viens du Sud, de très loin, peut-être d'un pays qui n'existe plus. Pour moi, il $n$ y a rien eu avant, juste cette rue poussiéreuse, l'oiseau noir, et le sac." (39)

L'héroïne de Le Clézio à cet âge, est un poisson sans identité complète, sans culture et sans voix. Elle quitte l'Afrique parce qu'elle a été volée à sa tribu des "Ouled Halil", le peuple au croissant de lune, qui vit dans le sud marocain dans la région de Foum Zguid. Vendue à une vieille dame, Lalla Asma qui est devenue sa maitresse, elle va faire son apprentissage dans la grande ville après avoir tout oublié de son enfance. " Seul souvenir, celui du rapt brutal, violent, inattendu, un grand sac qui se referme sur elle et le cri déchirant d'un oiseau noir qui marque le moment décisif de son existence où elle a été dépossédée de son identité. Car c'est cela l'histoire de Laila. A travers toutes ses aventures, ses tribulations, ${ }^{\prime \prime(40)}$ ses exils en France ou aux Etats-Unis, est une quête à la recherche de sa culture et son identité, car personne ne peut vivre sans racines. Comme dans un conte initiatique, l'héroïne va devoir partir, subir de nombreuses épreuves pour réparer les lacunes.

Par conséquent, l'espace géographique qui sépare les cultures et les races disparait, tandis que la terre d'adoption devient familière par son caractère universel qui permet à chacun de trouver son propre espace, la terre natale est lointaine non seulement du point de vue géographique, mais aussi émotionnel. L'héroïne préfère garder son souvenir que de confronter la vision de la réminiscence avec la réalité. C'est-à-dire qu'un lecteur étranger 
introduira dans la réception de l'œuvre une série de sens qui ne pouvaient être imaginés par l'auteur. La conclusion de Bakhtine nous conduit " au fait que la culture ne fait que s'enrichir au travers d'un regard autre donc dialogiquement, d'où l'importance du phénomène exotique dans le cas de la réception: "Dans le domaine de la culture, l'exotopie est le moteur le plus puissant de la compréhension. Une culture étrangère ne se révèle dans sa complétude et dans sa profondeur qu'au regard d'une autre culture"(4I)

" La rue du Javel, c'était l'endroit le plus extraordinaire de Paris. D'abord, je ne voulais pas croire que ça existait. (...) Mais la rue tournait sous la terre, dans une galerie bétonnée, avec les portes des garages, et le bruit de la moto résonnait comme l'enfer." (42)

En tant que tels, nous sommes des sujets hybrides ou "impurs", des composés d'esprit et de matière, de conscience et de matérialité, d'identité et d'altérité, selon Bakhtine qui "cherche à faire passer l'ontologie du terrain du système abstraits à celui de ce monde que nous partageons et qui est le sol essentiel de l'expérience et de l'action humaine. Les êtres humains ont une conscience corporelle pré-réflexive du monde et ils sont situés de manière unique et inextricable dans un espace-temps concret." (43) Ainsi, l'écrivain ne s'arrête pas pour nous décrire l'urbanisme de ces villes, mais présente les cultures de ces villes, les souvenirs de quelques lieux et éléments de décor:

" J'ai attendu longtemps sous la galerie, en concentrant mon attention sur le va-et-vient des marchands dans la cour. Je n'avais rien mangé depuis la veille, j'avais très faim, et je mourais de soif....

Pour s'identifier avec l'autre, il y a deux éléments encouragent l'héroïne dans Poisson d'or: l'aliénation initiale par rapport à soimême et l'aliénation postérieure par rapport à l'autre. Tout commence chez l'héroïne par le sentiment d'étrangéité avec la terre natale. Le démarrage de la terre natale commence avec la question; " Qui suisje?, avec la recherche de sa propre identité. La terre inconnue pousse l'exilée au sentiment de solitude et d'aliénation. Dans ce sentiment d'étrangeté nait la conscience de l'appartenance à un groupe au-delà des limites établies par des repères géographiques. Ce groupe est relié par un sentiment de solitude qui réside à l'intérieur de soi et qui est satisfait, par la création artistique. Par la suite, il y a un autre aspect de l'exil de l'héroïne, qui n'est plus solitaire, mais au contraire engagé dans la foule de rencontres multiculturelles et multinationales. Dans la dernière étape de l'exil clézien, l'héroïne est entourée des gens, des amis ou des connaissances passagères. L'exil volontaire est une attitude négative de l'identité homogène. L'héroïne critique l'Afrique 
à cause de sa démécanisation, ce qui rend cette terre complétement hostile à la nature humaine:

" J'ai eu du mal à trouver la maison de Tagadirt. Le Douar Tabriket était loin du fleuve, dans un quartier en hauteur, fermé par une grande route en construction où roulaient les camions. C'était très pauvre, rien que des baraques de planches couvertes de plaques de tôle ou de fibrociment calées par des pierres pour résister au vent. Les rues étaient toutes pareilles, des allées de terre bien droites tourbillonnantes de poussière. La grand-route faisait un encore plus grand nuage rougeâtre au-dessus de la ville. "(45)

Le langage utilisé par G. Le Clézio dans le passage ci-dessus se caractérise par l'accumulation d'adjectifs péjoratifs: "mal", " fermé", " grande route", "très pauvre", pareilles", " bien droites" loin de fleuve". A côté de la forte valeur qualitative de sa description, Le Clézio la rend dynamique par l'emploi fréquent des exclamations et des phrases nominales ou adverbiales, ce qui accélère le rythme du langage. Le lecteur rencontre l'héroïne rarement seule. En fait, ces rencontres multiples et multinationales soulignent le caractère unique de chaque personnage et une certaine impossibilité de fuir la solitude, qui nous accompagne aussi bien avec l'autre qu'en soi-même. Mais l'effet de la rapidité décrite est rendu par la description elle-même. Cette rapidité de la vie à Paris est vue comme positive à l'héroïne qui échappe aussi au piège du sentiment idéalisant, propre aux émigrants:

" Nous avons pu dormir dans la camionnette qui nous emportait vers Toulouse.au point du jour, elle était sur la route, au bout du bois, et l'Espagnol nous a fait monter très vite. Puis il est reparti vers la montagne, sans même un regard ou un signe d'adieu." (46)

C'est-à-dire que le conflit identitaire d'un être vivant sur la frontière entre le national et l'universellement humain se multiplie, au-delà de toute division nationale, d'héroïne-flâneuse dans la ville multiculturelle de Paris qui est une ville pleine de différentes cultures et, en même temps, le refus de l'unité culturelle. Cette multiplicité culturelle sert dans Poisson d'or à tracer un "moi multiple", qui se cherche à travers le regard des autres et sur les autres.

" Je marche pendant des jours. Jusqu'au bout des rues, jusqu'à la mer. Jusqu'au bout du monde, jusqu'à la mort. Je glisse entre les gens, entre les voitures, je cours souvent.je suis la plus rapide. Rien ne peut m'arrêter. J'ai appris à courir il y a longtemps, quand je suis sortie de la cour de Lalla Asma. J'ai appris à éviter les pièges, (....) Le vent cogne mon visage, je sens l'odeur de leurs dix pneus qui lèvent en roulant une fine poussière noire." " (47) 
Ce passage présente bien le refus du retour au pays natal. Les moments d'émotion sont considérés comme une faiblesse et ils sont immédiatement suivis par des moments de lucidité. Il y a chez l'héroïne la conscience de la coïncidence entre l'abstrait et le réel. L'exil met en scène une autre localisation incarnant l'absence et l'écart, présents seulement dans la mémoire de l'héroïne qui forme son identité. Cet espace est la terre natale, qui, bien que rejetée, sert au moi narratif de point de référence. La France, sa culture et sa structure sociale seront confrontées et opposées à l'Afrique dans la mémoire de l'héroïne.

L'espace de la mémoire se trouve bien défini dans Poisson d'or. La narratrice y reconstruit sa vie en se remémorant les événements-clés qui ont marqué sa jeunesse et son âge adulte, par exemple tous les déplacements entre les deux pays; La France et Le Maroc, qu'elle a habités. Il faut cependant signaler les limites imposées par la mémoire. C'est justement à Le Clézio que G. Poulet a consacré " une reflation sur l'espace qui est la répondante des études sur les temps humains. " (48) C'est la mémoire de l'héroïne qui est une activité par laquelle passe dans l'esprit la conservation et le retour d'une idée antérieurement acquise. Nous pourrions dire que l'espace de la mémoire est une rédaction des faits qui sont passés durant la vie de la narratrice et dans lesquels elle a joué un rôle. Pouvons-nous utiliser la notion de " lieu de mémoire " développée dans l'œuvre fondatrice dirigée par Pierre Nora, qui offre un cadre conceptuel adéquat pour l'analyse des relations entre espace et identité, selon lui : "les lieux de mémoire sont lieux dans les trois sens du mot, matériel, symbolique et fonctionnel, mais simultanément, à des degrés seulement divers." ${ }^{(49)}$ La mémoire de l'hérö̈ne est une connaissance immense, elle peut relater l'Afrique en français par un instrument d'optique destiné à l'observation des personnages et des objets très éloignés, l'Afrique porte le sens symbolique pour l'héroïne qui éprouve un sentiment d'appartenance, au contraire de Paris qui est le lieu préféré de l'héroïne portant l'idée de patrimoine et le sens matériel, cette expression qui " n'est pas seulement associée à la règle de l'héritage, elle est liée à la perpétuité d'objets sacrés essentiels à la communauté. " ${ }^{(50)}$ Les mouvements qui touchent Paris sont des éléments du renouveau de l'identité chez l'héroïne, où Paris est le berceau de tous ses songes, ses rêves éveillés, ses visions, ses opinions les plus profondes. Paris est ce lieu qui ne saurait disparaître.

En effet, la narratrice réalise une vision rétrospective sur sa vie, étant à l'origine de toutes ces questions qui la font réfléchir sur son identité, son espace et sa voix. Le" Je" de la narratrice révèle son espace intérieur, tel un regard intime. Dans l'espace du souvenir, peut-on dire que la rue blanche et l'oiseau noir animent la mémoire de la narratrice? A travers son circuit au Sud, l'héroïne entend monter les voix qui expriment le malheur du pauvre. De ces souvenirs, on connaît plus de détails, plus précis à la fin de Poisson d'or. 
Les noms de rues par exemple, qui l'exaltaient en lui rappelant un passé illustre ; Avenues de Paris, le chemin de retour, la ruelle, le fondouk,......etc. C'est la disposition de l'espace qui concrétise des scènes de la vie sociale des oubliés. Une mémoire à multiples fonds de la narratrice crée un personnage omniprésent. Elle n'a pas une voix comme les autres, mais elle a une mémoire polyphonique qui pousse l'héroïne à éviter la peur :

"Maintenant, je n'avais plus peur des mêmes choses. Je pouvais regarder les gens droit dans les yeux et leur mentir, même les affronter. Je pouvais lire leurs pensées dans leurs yeux, les deviner, et répondre avant qu'ils aient eu le temps de poser une question. Je pouvais même aboyer, comme ils savent si bien le faire ${ }^{(51)}$

Dans cette citation, on remarque la stabilité de l'identité de "Laila" qui affronte les difficultés sans peur, sans hésitation, cette femme devient un personnage audace, actif, prudent et elle peut lire les pensées des gens à travers leurs regards.

Ajoutons que l'espace de la mémoire se trouve bien défini dans Poisson d'or, quand l'héroïne reconstruit sa vie en se rappelant les actes clés qui ont marqué sa jeunesse et son âge adulte en Afrique et en France. Il faut néanmoins annoncer les limites imposées par la mémoire. En exprimant ses souvenirs, bons et mauvais, en rassemblant les segments de son passé, elle témoigne du déracinement, de l'immigration, de la nostalgie et de l'exil. Dans ce texte, nous pouvons remarquer une ouvre multiple, à plusieurs tiroirs et hybride. Ce genre de texte correspond à " un large éventail de critères génériques. " (52)

La situation de l'héroïne clézienne est très particulière; c'est une exilée africaine à Paris, une "autre" dans une société multiculturelle et hybride. L'héroïne est une étrangère et la plupart de ceux qu'elle rencontre sont des étrangers: juifs, allemands, arabes, ...etc. La description de ces différentes images présente la proximité des différentes nationalités sur un même espace absolument petit. D'ailleurs, l'exiguïté de l'espace est une des problématiques essentielles présentées dans "Poisson d'or". Tous vivent les uns à côté des autres, les dialectes se mélangent dans un bruit mystérieux des cafés et des rues, qui se croisent dans une planimétrie ouverte. Le Clézio situe toute la trame de "poisson d'or" dans la rue: toute rencontre se passe dans la rue ou plutôt dans la multitude des rues. L'instabilité dans la prose de Le Clézio est généralement solitaire, l'héroïne sort dehors toute seule et vagabonde sans un but déterminé. La rue est décrite comme une stimulation au travail créatif de la mémoire, si importante dans l'écriture autobiographique.

Certains noms de lieux peuvent être significatifs. Ces noms pourront figurer dans l'une ou l'autre catégorie selon le point de vue où l'on se place. Sur un total à peine supérieur à plusieurs onomastiques et toponymies, Le 
Clézio a utilisé ces noms de lieux pour présenter au lecteur ce que ce Poisson d'or renferme de réel et d'imaginaire. Depuis le début de l'œuvre, il a cité ces toponymies comme des noms de pays étrangers, des noms de villes, des rues, des quartiers ....etc. Nous avons ainsi remarqué que les références données portent essentiellement sur des villes faisant partie du patrimoine culturel de Paris. L'auteur présente les souvenirs de quelques lieux et éléments de décor comme "Les rues de Paris me semblaient sans fin." (53) en effet, Paris sans fin par ses avenues, les anciens musées, aussi, les anciennes églises, ses immeubles, ses rues bordées de jasmin ... la narratrice croit que si elle voulait parcourir toutes les rues, l'une après l'autre, sa vie n'y suffirait pas. Ici, le référent spatial est mis au service du sens dans la mesure où " le réseau des relations auquel appartient le personnage s'étend aussi aux lieux et aux objets. "(54) La diversité des lieux réels présents dans les textes attire de prime abord notre attention. Ces lieux s'attachent toujours au temps du personnage car : "l'espace qu'il soit « réel » ou «imaginaire », se trouve (...) associé, voire intégré aux personnages, comme il l'est à l'action ou à l'écoulement du temps. "(55)

L'existence entre deux pays est la véritable source de l'écriture et elle porte un sentiment nostalgique au bon vieux temps de l'enfance. Mais quel but l'auteur cherche-t-il à atteindre en mentionnant ces différents toponymes dans son Poisson d'or? Greimas et Courtes définissent ces toponymes comme étant:" des noms qui permettent un ancrage historique (un peu à la façon du personnage référentiel historique), visant à construire le simulacre d'un référent externe et à produire l'effet de sens réalité." ${ }^{(56)}$ Ce qui importe ici ne sont pas les noms de lieu en eux-mêmes, c'est leur valeur. Des noms produisent certains effets et surtout ils nous renseignent sur l'univers imaginaire de Le Clézio. Ajoutons que la présence de ces toponymes référentiels peut avoir deux valeurs chez Le Clézio : ces lieux aménagent la fiction dans le réel et ils mettent des relations étroites avec les personnages: " Les Africains jouaient du tambour, de grands tambours de bois couverts de peau, très doucement, du bout des doigts, et à la lumière des bougies, les garçons dansaient.

Le Clézio a inscrit la fiction dans le vraisemblable en utilisant des toponymes ayant pour référents des espaces réels. Il a essayé de nous convaincre que le texte mis sous nos yeux, appartient à la réalité. Le Clézio ne s'intéresse pas à donner d'emblée au lecteur des descriptions détaillées, au contraire des romanciers réalistes et naturalistes, ces descriptions détaillées sont le plus souvent considérées " comme parasites." (58) C'est-à-dire que l'espace contribue à la définition du personnage parce qu'il décrit son milieu. Paris, espace de l'exil, devient aussi l'espace d'une transformation, d'une initiation du héros de l'âge adulte à l'âge de la maturité, par le biais de 
l'amour, de la mort et de la maladie. Espace paradisiaque, tout comme le temps de l'enfance:

"Paris, au début, c'était magnifique. Je courais les rues, je n'arrêtais pas. Houriya, elle, restait enfermée dans le meublé, elle faisait la cuisine, elle observait. Elle avait peur de tout. Comme autrefois au fondouk, c'était moi qui faisait les courses, qui allais partout......" (59)

Le Paris clézien est à la fois formidable et resserré; ces deux aspects résultent de la multitude des rues et des visages. C'est un endroit à plusieurs façades, souvent contradictoires. Si d'un côté il ressemble à une terre nouvelle, découverte par une exilée africaine provenant d'une réalité différente, de l'autre, il représente un endroit qui inspire une étrange impression de familiarité. C'est à Paris que l'héroïne trouve son identité, là elle ressent son appartenance à un groupe, le groupe des artistes. Ce caractère de l'espace illimité par la multitude de rues et de rencontres la pousse vers une nouvelle découverte. La naissance de l'expérience à Paris étant simplement une scène artificielle, un plateau tournant qui permet au spectateur d'apercevoir toutes les durées du conflit. En effet, Paris est le berceau des naissances artificielles. Doucement, tout le monde y a vécu, à un moment ou un autre. Elle cite la description de Paris en disant:

" Les rues de Paris me semblaient sans fin. Et certaines étaient réellement sans fin, avenues, boulevards qui se perdaient dans le flux des autos, qui disparaissaient entre les immeubles. Pour moi qui n'avais connu que le monde du Mellah et le bidonville de Tabriket, ou les petites rues bordées de jasmin du quartier de l'Océan, cette ville était immense, inépuisable.je pensais que même si je voulais parcourir toutes les rues, l'une après l'autre, ma vie n'y suffirait pas. Je ne pourrais voir qu'une petite partie, un nombre restreint de visages ${ }^{(60) !}$

Paris est une ville d'immigrants d'origine européenne ou arabe. Toutefois, au cours du $\mathrm{XX}^{\mathrm{e}}$ siècle, les origines culturelles des immigrants se sont grandement diversifiées. A vrai dire, cette pluralité culturelle change désormais la composition de la population parisienne. A Paris, les écrivains immigrants cherchent à se définir dans leur nouvel espace géographique, culturel et linguistique. Jean Weisgerber définit l'espace du roman comme : " un ensemble de relations existant entre les lieux, le milieu, le décor de l'action et les personnages que celle-ci présuppose, à savoir l'individu qui raconte les événements et les gens qui y prennent part." "(61) Paris n'est pas un abri tranquille pour l'artiste ou pour l'écrivain, mais dans sa représentation de la ville, le lecteur retrouve que Paris est illimité et éternel. Le lecteur peut découvrir que Paris, elle-même, est un cancer: 
"J'ai continué d'explorer paris, tout l'été. Il faisait un temps magnifique, un ciel bleu sans un nuage, les arbres étaient encore très verts, brillants. Les orages d'aout avaient grossi la Seine. (......) je marchais dans tous ces quartiers: Bastille, FaidherbeChaligny, la Chaussée-d'Antin, L'Opéra, la Madeleine, SaintAntoine, Saint-Paul.il y avait des quartiers bourgeois, élégants, qui dormaient à trois heures de l'après-midi, des quartiers populaires, des quartiers bruyants, de longs murs de brique rouge pareils à l'enceinte d'une prison... ${ }^{(62)} . . . "$

L'image du cancer présentée dans les passages de Poisson d'or, est marquée par la violence, c'est une image de l'animal dégoutant et effrayant qui ne cesse pas de grandir. Par l'écoulement du temps et l'invasion de l'espace se traduit la vision terrible de la narratrice clézienne. Paris exposé par la narratrice n'est pas éternel par sa beauté, mais par la souffrance qu'il inflige. La narratrice chez Le Clézio insiste souvent sur l'aspect de la souffrance vécue dans cette ville. Paris personnifie une accumulation, tout un vécu de sentiments, d'émotions et de pensées de trois étapes distinctes dans la vie de l'héroïne, que le lecteur découvre, soit à travers le présent de la narration soit par les souvenirs de l'héroïne ou le passé de la narration.

Le lecteur a l'impression de visiter un endroit rempli de rencontres et de conversations bourdonnantes dans la multitude des langues. Les nombreuses nationalités qui peuplent la ville, la rendent immense et illimitée par sa pluralité et sa variété. Ce "multiculturalisme" parisien est rendu par l'usage de plusieurs hétéro-images; Paris attire des touristes, des aventuriers et de pauvres immigrés qui fuient leur propre pays. Par la présentation de différents imagotypes, la narratrice dans ce roman brosse au lecteur un Paris croisé et désordonné. C'est un tableau de la pluralité au niveau social, mais aussi au niveau de la structure des personnages du Poisson d'or, c'est une peinture de la nature humaine dans sa complexité. Les personnages du roman se caractérisent par la nature pleine de contraste; ce sont des êtres capables de réflexion profonde et humaine.

Ce multiculturalisme influe sur l'identité de l'héroïne qui hésite entre deux mondes; l'orient et l'occident, entre les traditions et la liberté, entre la logique et l'irrationnel. Dans ce travail, on examine divers aspects des ambivalences identitaires engendrées par la ville et leur représentation romanesque chez Le Clézio.

C'est-ainsi que, Laila qui est comme le poisson, le symbole de l'âme résistante à ce monde puisqu'elle peut non seulement pousser dans cet espace caractérisé par la sécheresse, la pauvreté et l'aridité, mais aussi par donner des fruits. Il y a toujours une source de vie, d'espoir et de sagesse et il faut rappeler que l'espace mis en scène par l'écrivain représente des étapes différentes du roman. Chaque lieu évoque " une certaine réalité sociale, 
l'ensemble donne l'impression de vie et de vérité." (63) L'espace dans Poisson d'or remplit ses fonctions primordiales qui consistent à conférer une plus grande authenticité à la réalité représentée dans ces textes, cet espace a souvent une valeur symbolique propre. L'espace a une influence sur le comportement des personnages, sur leur culture, sur leur pensée et sur leur identité. La relation d'un personnage avec un cadre donné peut développer une thématique ou donner des indices sur la vraie nature de ce personnage. L'écrivain place les personnages en fonction du sens qu'il veut produire. Donc, l'espace joue un rôle très important, il est exprimé par la manifestation de flânerie dans un endroit nouveau. Cet endroit, nous l'avons vu aussi, étant ignoré donne l'émotion de l'infinité par la multitude de rues qui se croisent dans la topographie sans fin, mais c'est un endroit qui relie profondément les unes aux autres et renferme plusieurs nationalités, lesquelles se côtoient sans cesse.

\section{Conclusion:}

Ce roman relate l'histoire d'une femme africaine en exil, une femme qui vit et explore non seulement un exil physique et géographique, mais aussi un exil intérieur, malgré le fait qu'elle se soit exilée volontairement à Paris, "n'est pas cependant littéralement l'exil."(64) C'est un roman d'apprentissage, un roman picaresque aussi, car Laila, ce poisson dans ses voyages continue à la recherche d'elle-même, va connaître bien des événements difficiles, douloureux parfois, va subir la faim, les manques, la peur, l'exploitation. L'héroïne sera obligée pour survivre d'utiliser toutes ses armes, de ne compter que sur elle-même. Poisson d'or présente un monde qui n'est pas tendre pour les pauvres, qui écrase les faibles. L'auteur offre son point de vue à travers une enfant. Mais derrière cette enfant," un autre point de vue, celui de la narratrice, plus âgée, qui corrige le point de vue de l'enfant ${ }^{(65)}$ Ce Poisson d'or devient un excellent espace où se croisent des souvenirs d'enfance divers, douloureux par les horreurs vécues ainsi que par l'impossibilité physique de rejoindre les temps révolus évoqués. L'écrivain a consacré une grande partie à une période historique pour faire revivre le passé à travers la figure de son enfance.

Poisson d'or a le sentiment d'être absorbé par le circuit du temps qui tourne dans un constant déplacement de souvenir, le reflet et la création de l'héroïne (le poisson) qui peut essayer des vertiges à cause des sauts continus entre le passé et le futur. A travers toutes ses aventures, ses tribulations, ses exils en France ou en Afrique, c'est une quête à la recherche de son origine et son identité, car personne ne peut vivre sans racines. L'exil volontaire est une attitude de négation de l'identité homogène; la terre natale parait comme un espace étrange. L'héroïne critique l'Afrique à cause de sa démécanisation, ce 
qui, d'après lui, rend cette terre absolument hostile à la nature humaine. Sa voix devient extérieure par rapport à sa communauté d'origine. Elle s'exile et s'est sortie de sa mer pour raconter une histoire qui parle de son pays dans une vision différente et cette vision donne à son histoire une dimension multinationale.

Enfin, Poisson d'or est délimité par les formalités spécifiques du temps et de l'espace, agrandis par l'action du voyage. Le Clézio ne décrit pas un déplacement dans un seul espace, mais dans plusieurs espaces, puis son Paris est une terre de proximité de distinctes nationalités. L'héroïne de Poisson d'or est une exilée africaine qui vit à Paris, cette ville qui attirait les individus d'origine et de cultures différentes. Paris. Elle est chargée d'une joie absurde, qui éclate devant la menace de la violence, mais aussi le désir d'oublier cette violence et de trouver une liberté propre afin de présenter son identité et sa culture. Par ces sentiments-là, le lecteur peut découvrir dans Poisson d'or: la folie, la joie sinistre, la nostalgie et la pensée sur l'avenir de l'humanité.

\section{I- Corpus de l'étude}

\section{Bibliographie}

(1)- LE CLEZIO (J.M.G), Poisson d'or, Paris, éd: Gallimard, 1997.

II- Ouvrages de la critique

(2)- BACHELARD (Gaston.), La poétique de l'espace, Paris, Puf, 1957.

(3)- $\quad$-La poétique de la rêverie, Paris, PUF, Quadrige, 5è édition.

(4)- BAKHTINE (Mikhail), La Poétique de Dostoïevski, trad. Du russe par Isabelle

Kolitcheff, présentation de Julia Kristeva, Moscou, Ecrivains soviétique, 1963, Paris, Seuil, 1970.

(5)- $\quad$ - Esthétique et théorie du roman, Gallimard, 1978

(6)-BENVENISTE (Emile), Problèmes de linguistique générale. Tome 11. Paris,

Ed : Gallimard. 1974.

(7)-BERGEZ (Daniel) et autres, Introduction aux méthodes critiques pour

l'analyse Littéraire, Edition : Mise à jour, DUNOD, Paris, 1999.

(8)- BOURNEUF (Roland) et OUELLE (Réal), L'univers du roman, Paris, PUF, Coll. «SUP », 1972.

(9) - CHASTEL (A), La notion de Patrimoine, Les Lieux de Mémoire, II La Nation,

(10)- COMBE (Dominique), Les genres Littéraires, Hachette, Paris, 1992.

(11)-DESSINGUE (Alexandre), Polyphonisme de Bakhtine à Ricour. Accessed December 27, 2012.

(12)- GREIMAS(A.J) et COURTES (J), Dictionnaire raisonné de la théorie du langage, Édition : Hachette, 1979, p. 133

(13)- GRIVEL (Charles), Production de l'intérêt romanesque - un état du texte(1870-1880), un essai de constitutions de sa théorie, Paris, Mouton, The Hague, 1973.

(14)- DOMINIQUE (Maingunau), Eléments de linguistique pour le texte littéraire.

Paris, Bordas, 1986.

(15)-NORA (P), Entre mémoire et histoire. La problématique des lieux, Les lieux de mémoire I, Édition : La République, Gallimard, 1984, p. XXXIV

(16)- POUILLON (J), Temps et Roman, Paris, Gallimard, 1946. 
(17)- REUTER (Yves), Introduction à l'analyse du roman, Paris, Bordas, 1991, rééd Dunon, 1996.

(18)- REY (Pierre- Louis), Le Roman, Édition : Hachette, Paris 1992

(19)- RICARDOU (Jean), Problèmes du nouveau roman, Édition : Seuil, Paris, 1967.

(20) -RICOEUR (Paul), Temps et récit II, La configuration du Temps dans le récit de fiction, Ed : Du Seuil, Paris VI, 1984. Coll." Point Essais "

(21)-THEURET (Françoise Rullier), Approche du roman, Ed : Hachette Livre, Paris, 2001.

(22)- WEISGERBE (Jean), L'espace romanesque, Lausanne, l'âge d'homme,

Coll. " Bibliothèque de Littérature comparée " 1978.

(23)-WELLEK et WARREN (Austin), La théorie littéraire, Ed : Du Seuil, Paris, 1971.

\section{III - COLLOQUES}

(24)- NABIL (Oussama), Colloque - dans son article Le problème du genre littérature à travers Trois contes de l'amour et de la mort d'Out-el-Koloub dans l'université Al-Azhar, Faculté des langues. Département de français, Le Caire, 2001. p.13.

\section{VI- THESES}

(25)- NABIL (Oussama), Thèse de doctorat, Le réel et l'imaginaire chez Taher Ben Jelloun, Université de L'Azhar- faculté de langues et de traduction, Le Caire, 1993.

(26)- TREMBLAY (Marie-Claude Bussieres), Une "création sur les voix" à partir de la théorie de la plurivocalité de Mikhail Bakhtine, université du Québec, mars 1990, dans le cadre du programme de maitrise en études littéraires.

\section{IV- INTERNET}

(27)-http://www.di.ens.fr/ granboul/enseignement/temps/histoire.

(28)-Critique par Claudialucia,http://wwwlecture-ecriture.com/fiche_membre.php92

(39)-http//www.fabula.org/atelier.php?polyphonisme de Bakhtine à Ricour.

(30)- Dina Kabil ; L'exil, lieu d'écriture

(31) http://hebdo.ahra,.org.eg/arab/ahram/2005/2/23/idees $2 . h t m$

(32)- http://www.uquebec.ca/puq/revue/-html

(33)-

harmattan.fr/auteurs/article pop.asp?no=10125\&no artiste $=16894$

https://www.editions-

(1) - BAKHTINE (Mikhail), La Poétique de Dostö̈evski, trad. Du russe par Isabelle Kolitcheff, présentation de Julia Kristeva, Moscou, Ecrivains soviétique, 1963, Paris, Seuil, 1970, p. 31

${ }^{2}$ (2) - DeSSIngue (A), Polyphonisme de Bakhtine à Ricour. Accessed December 27, 2012. pp. 13,14

http//www.fabula.org/atelier.php?polyphonisme de Bakhtine à Ricœur.

(3) - BAKHTINE (Mikhaïl), Esthétique et théorie du roman, Gallimard, 1978.

(4) - BACHELAR (G.), La poétique de l'espace, Paris, Puf, 1957.

(5) - HAMON (Philippe), Le Roman des théories aux analyses, Texte et Idéologie, Ed : PUF, Ecriture, 1984

$\left({ }^{6}\right)$ - BAKHTINE (Mikhaï), op.cit. pp. 46, 47.

(7) - TREMBLAY(Marie-Claude Bussieres), Une "création sur les voix" à partir de la théorie de la plurivocalité de Mikhail Bakhtine, université du Québec, mars 1990, dans le cadre du programme de maitrise en études littéraires, p. 8 
(8) - CHARLES (Grivel), Production de l'intérêt romanesque - un état du texte (18701880), un essai de constitutions de sa théorie, Paris, Mouton, The Hague, 1973. p. 102 (9)-DESSINGUE (A), op. cit, p. 10

(10) - WELLEK \& WARREN (Austin), La théorie littéraire, Ed : Du Seuil, Paris, 1971, p. 35

(11) - cf. NJEUKAM Nouago Njeukam, l'espace et le temps romanesques: deux paramètres poétiques de lisibilité de l'échec de la quête de la modernité dans l'avenenture amibiguée de cheikh Kamidou Kane, [consulté 01 mar 2019], disponible sur Internet <URL: https://www.editions-harmattan.fr/auteurs/article pop.asp?no=10125\&no artiste $=16894$

$\left({ }^{12}\right)$ - Ricoeur. Paul, Le Temps et récit II, Ed : Du Seuil, Paris, 1984, p. 120

$\left({ }^{13}\right)$ - RULLIER (Theuret. Françoise), Approche du roman, Ed : Hachette Livre, Paris, 2001, p. 61

$\left({ }^{14}\right)$ - Le Clézio (J.M.G), Poisson d'or, éd: Gallimard, 1997, p. 11

$\left({ }^{15}\right)$ - RULLIER (Theuret. Françoise), op. cit, p. 69

$\left({ }^{16}\right)$ - Poisson d'or, op. cit p. 28

$\left({ }^{17}\right)$ - Ibid. p. 28

(18)- Ibid. p. 29

(19) - Ibid. p:252

(20)-REUTER(Yves), Introduction à l'analyse du roman, Paris, Bordas, 1991, rééd Dunon, 1996. p. 134

(21) - Poisson d'or, op.cit p. 46

(22) - Ibid. p. 133

( ${ }^{23}$ )- BENVENISTE (Emile), Problèmes de linguistique générale. Tome 11. Paris, Ed:Gallimard. 1974, p.70

$\left({ }^{24}\right)$ - DOMINIQUE (Maingunau), Eléments de linguistique pour le texte littéraire. Paris, Bordas, 1986, p. 22

$\left({ }^{25}\right)$ - Poisson d'or, op.cit, pp. 72, 73

$\left({ }^{26}\right)$ - Ibid., p. 141

$\left({ }^{27}\right)$ - RULLIER (Theuret. Françoise), op. cit, p. 71

(28) -GRIVEL(Charles), Production de l'intérêt romanesque - un état du texte (1870-

1880), un essai de constitutions de sa théorie, Paris, Mouton, The Hague, 1973, p. 101

$\left({ }^{29}\right)$ - Poisson d'or, op.cit. p. 179

$\left({ }^{30}\right)$ - NABIL(Oussama), Colloque, Le problème du genre littéraire à travers trois contes de

l'amour et de la Mort d'Out- el-Kouloub. Le Caire 2001, p. 29

(31)-Poisson d'or, op.cit. p. 132

(32)- FANON (Frantz), Les Damnés de la Terre, éd. La Découverte poche, 2002, p. 47-48

(33) - Poisson d'or, op.cit, p. 206.

$\left({ }^{34}\right)$ - BACHELARD (Gaston), La poétique de la rêverie, Paris, PUF, Quadrige, 5è édition, p.88.

$\left.{ }^{35}\right)$-Poisson d'or, op.cit, p. 179

${ }^{(36}$ ) -GRIVEL (Ch), Production à l'intérêt romanesque - Un état du texte, op.cit, p. 102

$\left({ }^{37}\right)$ - Poisson d'or, op.cit, p. 251

$\left({ }^{38}\right)$ - REY. (P. L), op. cit, p. 170

(39)- Poisson d'or, op.cit, p. 11

(40) - Critique par Claudialucia, http://wwwlecture-ecriture.com/fiche_membre.php 92?

(41)- BAKHTINE, op. cit. P.348

(42)- Poisson d'or, op.cit, p: 123

$\left({ }^{43}\right)$-Michel E. Gardiner, Le défi dialogique de Bakhtine aux sciences sociales, p: 83

$\left({ }^{44}\right)$ - Poisson d'or, op.cit p:31 

$\left({ }^{45}\right)$ - Ibid. p. 67
$\left({ }^{46}\right)$ - Ibid. p. 89
$\left({ }^{47}\right)$ - Ibid. P. 236
$\left({ }^{48}\right)$ - BERGEZ(D) et autres, sous la direction de Lui, Introduction aux méthodes critiques pour l'analyse littéraire, Édition mise à jour, Dunod, Paris, 1999, p. 111 $\left({ }^{49}\right)$ - NORA (P), Entre mémoire et histoire. La problématique des lieux, Les lieux de mémoire I, Édition : La République, Gallimard, 1984, p. XXXIV
$\left({ }^{50}\right)$ - CHASTEL (A), La notion de Patrimoine, Les Lieux de Mémoire, II La Nation, p. 247
$\left({ }^{51}\right)$ - Poisson d'or, op.cit, p:224
${ }^{52}$ - COMBE (D), Les genres littéraires, Édition Hachette, Paris, 1992, p. 153
$\left({ }^{53}\right)$ - Poisson d'or, op.cit, p. 97
$\left({ }^{54}\right)$ - BOURNRUF $(\mathrm{R})$ et OUELLE $(\mathrm{R})$, L'univers du roman, Paris, PUF, Coll. « SUP », p151
$\left({ }^{55}\right)$ - Ibid., p. 104
$\left({ }^{56}\right)$ - GREIMAS (A. J) et COURTES (J), Dictionnaire raisonné de la théorie du langage, Édition: Hachette, 1979, p. 133
$\left({ }^{57}\right)$ - Poisson d'or, op.cit, p. 102
$\left({ }^{58}\right)$ - BOURNEUF(R) et REAL.(O), op. cit, p. 109
$\left({ }^{59}\right)$ - Poisson d'or, op.cit, p. 95
$\left({ }^{60}\right)$ - Ibid. pp. 97,98
$\left({ }^{61}\right)$ - WEISGERBER(J), L'espace romanesque, Lausanne, l'âge d'homme, Coll." Bibliothèque de Littérature comparée" 1978, p. 14
$\left({ }^{62}\right)$ - Poisson d'or, op.cit, pp. 103, 104
$\left({ }^{63}\right)$ - Nabil. Oussama, Thèse de doctorat, op. cit, p. 122
$\left({ }^{64}\right)$ - AHRAM.HEBDO, Article de L'exil, lieu d'écriture, à 23-2- 2005. http := hebdo. Ahram. Org. eg /arab / ahram/
$\left({ }^{65}\right)$ - Critique par Claudialucia [consulté 12 mars 2019], disponible sur Internet ,http://wwwlecture-ecriture.com/fiche membre.php 92? 